\title{
Preoperative Evaluation of Paranasal Computed Tomography Reports of Patients Requesting Rhinoplasty for the Presence of Septal Deviation and Inferior Turbinate Hypertrophy: Retrospective Clinical Case Series Study
}

\begin{abstract}
Aksoy MH*
Department of Plastic, Reconstructive and Aesthetic Surgery, Bahcesehir University, Turkey

*Correspondling author: Hasan Mete Aksoy, Department of Plastic, Reconstructive and Aesthetic Surgery, Bahcesehir University, Istanbul; VM Medical Park Kocaeli Hospital, Plastic, Reconstructive and Aesthetic Surgery Clinic, Kocaeli, Turkey
\end{abstract}

Received: December 06, 2018; Accepted: J anuary 22, 2019; Published: J anuary 29, 2019

\begin{abstract}
Background: A successful outcome in rhinoplasty should improve not only the aesthetic appearance of the nose but also the physiologic function of the nasal cavity. Nasal obstruction in many patients is caused by a deviated nasal septum and/or inferior turbinate hypertrophy. Preoperative proper diagnosis of these conditions is very important in planning cosmetic nasal surgery.
\end{abstract}

Methods: Paranasal computed tomography reports of 119 patients requesting cosmetic nasal surgery were evaluated for the presence of septal deviation and inferior turbinate hypertrophy.

Results: In 62 patients (52.10\%) there was septal deviation with concave side facing the left nasal cavity and in 46 patients (38.65\%) there was septal deviation with concave side facing the right nasal cavity. In only 11 patients $(9.24 \%)$ there was no finding suggesting septal deviation. In 113 patients $(94.96 \%)$ there was inferior turbinate hypertrophy. In only 6 patients $(5.04 \%)$ there was no sign of inferior turbinate hypertrophy on paranasal computed tomography scan.

Conclusion: Despite the fact that radiologic imaging is usually not a standard part of the workup in patients who are candidates for rhinoplasty, preoperative paranasal computed tomography is essential to detect the presence of concomitant pathologies like septal deformities, inferior turbinate enlargement, bullous middle turbinate and chronic sinusitis. Preoperative paranasal computed tomography is a very valuable method to assess internal nasal structures especially for plastic surgeons who do not have endoscopic instruments to examine nasal cavities.

Keywords: Computed tomography; Paranasal; Septal deviation; Turbinate hypertrophy

\section{Introduction}

The role of nasal physiology should be well understood and respected by one who performs cosmetic nasal surgery. A successful outcome in rhinoplasty should improve not only the aesthetic appearance of the nose but also the physiologic function of the nasal cavity. Nasal obstruction in many patients is commonly due to a deviated nasal septum and/or inferior turbinate hypertrophy. Preoperative proper diagnosis of these conditions is very important in planning aesthetic nasal surgery. In this study paranasal computed tomography reports of patients who were candidates for cosmetic rhinoplasty operation were evaluated to detect the presence of septal deviation and inferior turbinate hypertrophy.

\section{Materials and Methods}

In this study paranasal computed tomography reports of 119

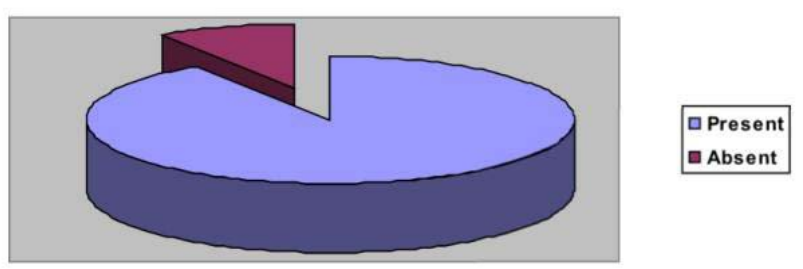

Figure 1: Diagram showing presence of septal deviation.

patients requesting cosmetic nasal surgery were evaluated for the presence of septal deviation and inferior turbinate hypertrophy retrospectively. Anterior rhinoscopy examination was performed in all patients prior to preoperative paranasal computed tomography. However, intranasal endoscopic examination was not performed since endoscopic instruments were not available at our plastic surgery
Austin J Surg - Volume 6 Issue 3 - 2019

ISSN : 2381-9030 | www.austinpublishing group.com

Aksoy. (C) All rights are reserved
Citation: Aksoy MH. Preoperative Evaluation of Paranasal Computed Tomography Reports of Patients Requesting Rhinoplasty for the Presence of Septal Deviation and Inferior Turbinate Hypertrophy: Retrospective Clinical Case Series Study. Austin J Surg. 2019; 6(3): 1163 


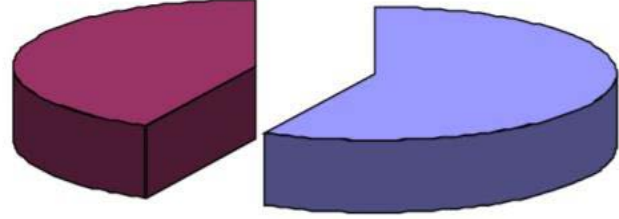

Figure 2: Diagram showing direction of septal deviation.

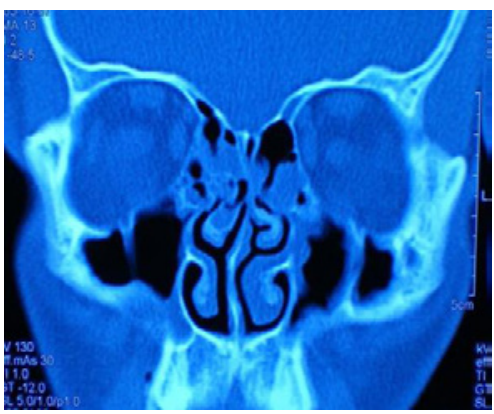

Figure 3: Paranasal computed tomography scan showing septal deviation with concave side facing the left nasal cavity. There is also hypertrophy of the left inferior turbinate.

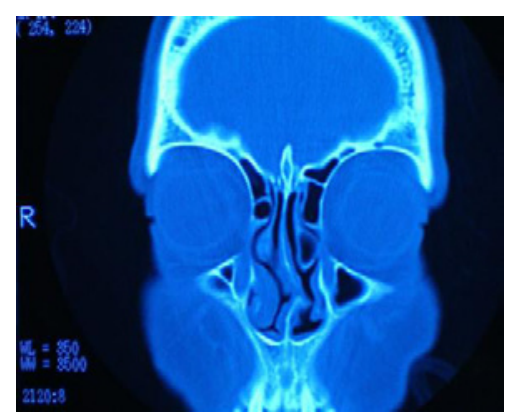

Figure 4: Paranasal computed tomography scan showing septal deviation with concave side facing the right nasal cavity. There is also hypertrophy of the right inferior turbinate and bullous middle turbinate is present in the left nasal cavity.

clinic. So obtaining a paranasal computed tomography scan prior to rhinoplasty was a routine practice in our clinic. This study was performed in accordance with Declaration of Helsinki.

\section{Results}

There were 47 males (39.4\%) and 72 females (60.5\%) in this study. The age range of the patients was 15-52 years and the mean age was 27.85 years. In only 11 patients $(9.24 \%)$ there was no finding suggesting septal deviation (Figure 1). In 62 patients (52.10\%), there was varying degrees of septal deviation with concave side facing the left nasal cavity (Figures $2 \& 3$ ) and in 46 patients (38.65\%), there was varying degrees of septal deviation with concave side facing the right nasal cavity (Figures $2 \& 4$ ). In 113 patients (94.96\%), there was varying degrees of inferior turbinate hypertrophy (Figures 5 \& 6). In only 6 patients (5.04\%) there was no sign of inferior turbinate hypertrophy on paranasal computed tomography scan (Figure 5). None of our patients had paranasal computed tomographic findings of chronic sinusitis requiring ENT consultation and surgical

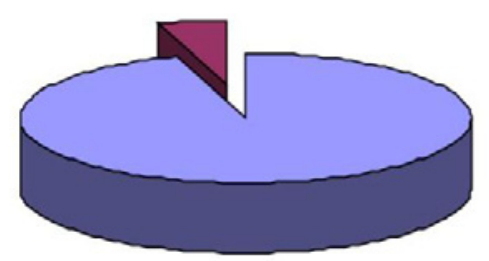

$\square$ Present

absent

Figure 5: Diagram showing presence of conchal hypertrophy.

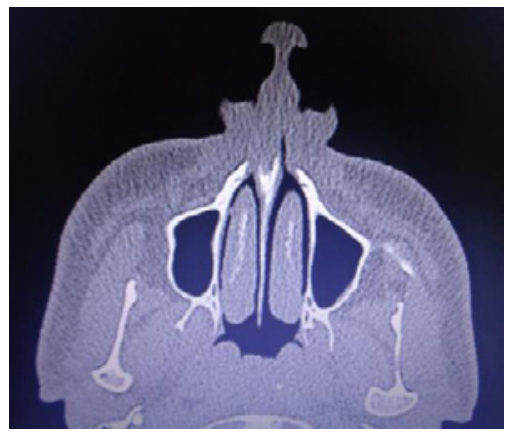

Figure 6: Paranasal computed tomography scan showing significant enlargement of both the left and right inferior turbinates.

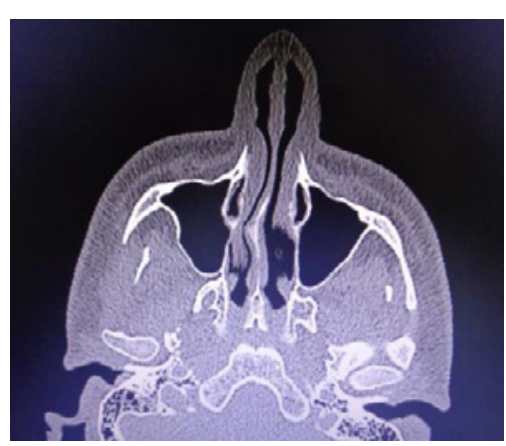

Figure 7: Preoperative paranasal computed tomography scan showing posterior bony nasal septal deviation.

treatment. Presence of bullous middle turbinate was not evaluated because bullous middle turbinates when detected were treated with the help of an ENT specialist during rhinoplasty procedure.

\section{Discussion}

Preoperative detailed structural evaluation and surgical management of nasal airway pathologies are essential in patients who are candidates for rhinoplasty. Unfortunately rhinoplasty is a frequent cause for litigation among elective facial aesthetic operations. Airway problems are the main concerns in some rhinoplasty cases following surgery. Nasal obstruction is associated with a remarkable decrease in quality of life of these cases. So detailed evaluation of nasal airway is very important prior to performing rhinoplasty.

Radiologic imaging is usually not a part of the standard workup in patients interested in rhinoplasty. Physical examination with anterior rhinoscopy and nasal endoscopy is considered the gold standard for evaluating nasal airways. Internal nasal examination is especially useful in detecting and rating septal deviations. ENT clinics have nasal endoscopic instruments but not all plastic surgery 


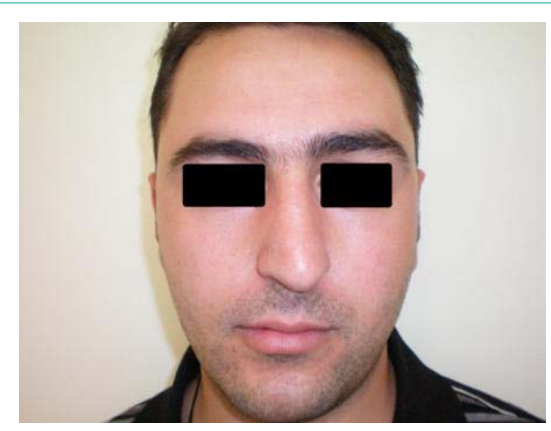

Figure 8: Preoperative picture of a patient with crooked or deviated nose.

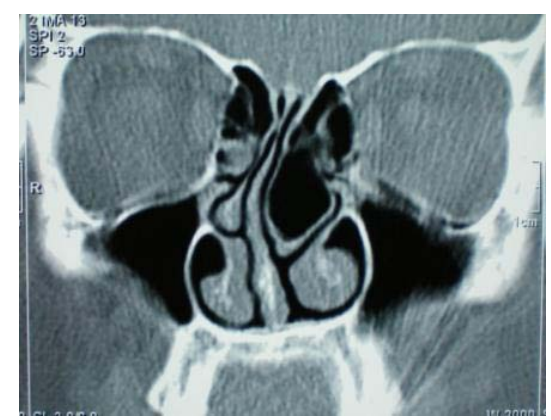

Figure 9: Paranasal computed tomography scan showing presence of a large bullous middle turbinate within the left nasal cavity.

clinics have nasal endoscopes. Availability of nasal endoscopic examination is a problem for plastic surgeons. In the absence of endoscopic examination radiologic imaging of nasal airways becomes important for plastic surgeons to evaluate intranasal pathologies prior to performing rhinoplasty. Paranasal computed tomography is a radiologic imaging method that can be used to evaluate nasal airways and nasal structures preoperatively. This radiologic imaging method can identify anatomic variations of the nasal bones and turbinates and helps planning nasal surgery correctly. This is important to prevent nasal airway problems following rhinoplasty. So obtaining a preoperative paranasal computed tomography scan becomes important if you don't have nasal endoscopic instruments. Despite the fact that you have nasal endoscopic instruments available for intranasal examination, preoperative paranasal computed tomography can be used for further examination of the nasal anatomy and identification of ancillary sinonasal pathologies [1]. So preoperative CT scan can identify pathologies not visible on physical examination, assist with surgical planning and help better relieve nasal obstruction. However this radiologic modality has additional costs and exposes patients to harmful radiation.

A preoperative paranasal computed tomography scan is very useful for diagnosis and evaluation of nasal obstruction and for surgical planning, because it reveals the middle and posterior nasal cavity (Figure 7), which can be overlooked by physical examination of the nose [2]. Thus radiologic imaging with paranasal computed tomography is important in evaluating nasal obstruction and this scan can be obtained following execution of anterior rhinoscopy and endoscopic evaluation of the nose. So when surgeons select patients for septal deviation surgery, they must use preoperative paranasal computed tomography and pay attention to the correction of middle

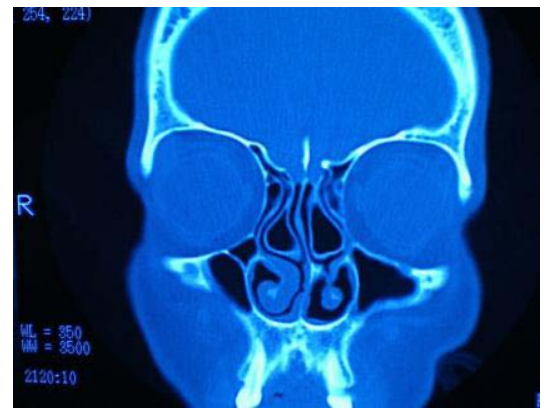

Figure 10: Paranasal computed tomography scan showing presence of large bullous middle turbinates within the left and right nasal cavities.

and posterior septal deviations [2].

Septal deviation is one of the main causes of nasal airway obstruction. Paranasal coronal and sagittal plane computed tomography was used for the detection and classification of nasal septal deformities $[3,4]$. In the majority of patients who requested cosmetic nasal surgery there were varying degrees of septal deviation in our study.

Crooked or deviated nose is a deviation of the nose from the straight vertical position of the face and preoperative paranasal computed tomography is essential for patients with a crooked nose even if they do not have concomitant sinonasal diseases like allergy and chronic sinusitis [5] (Figure 8).

Turbinates or conchae are important structures arising from the lateral wall of the nose. There are normally three turbinates. These are superior, middle and inferior turbinates. Occasionally there may be a fourth one called the supreme turbinate [6]. Most of the inhaled airflow travels between the inferior and middle turbinates.

The inferior turbinate is the largest turbinate. Inferior turbinates are responsible for the majority of airflow direction, humidification, heating, and filtering of air inhaled through the nose. Large and swollen inferior turbinates may cause blockage of nasal breathing. Usually enlarged inferior turbinates are the result of infections, allergies, exposure to irritants, such as cigarette smoking, vasomotor rhinitis and chronic infection in the paranasal sinuses [6]. Paranasal computed tomography can be used to evaluate enlarged inferior turbinates in detail and this radiologic evaluation makes it easier to understand the contribution of enlarged inferior turbinates (especially posterior parts) to nasal obstruction [2]. In the majority of patients who requested cosmetic nasal surgery there were varying degrees of inferior turbinate hypertrophy in our study.

A deviated nasal septum may lead to compensatory enlargement of the contralateral inferior turbinate [6,7]. We also observed this compensatory enlargement of inferior turbinates in patients with nasal septal deviation in our study. In a study performed by Jun et al paranasal computed tomography scanning showed that the inferior turbinate on the concave side of septal deviation had a significantly greater volume, including the thickness of medial mucosa and thickness and projection angle of conchal bone [7]. So septoplasty and concomitant inferior turbinate surgery to manipulate conchal bone and soft tissues are necessary for the treatment of patients with unilateral nasal septal deviation and compensatory hypertrophy of 
the contralateral inferior turbinate [7].

It is very unusual for enlargement of the inferior turbinate to be due to an inferior concha bullosa. Inferior concha bullosa is normally asymptomatic and diagnosed incidentally upon imaging [6]. However when there is extensive pneumatization, it can lead to nasal obstruction [6]. Other symptoms that may be associated with inferior concha bullosa are nasal discharge, nasal discomfort with coexisting infection, headache and epiphora [6]. Clinically, it is almost impossible to differentiate between hypertrophy of the inferior turbinate and an inferior concha bullosa [6]. Use of vasoconstrictor medicines may be useful in the clinical setting to differentiate between a concha bullosa and reversible nasal mucosa disease [6]. Definitive diagnosis is made with a paranasal computed tomography scanning [6]. We did not encounter any case with inferior concha bullosa in our study.

Middle turbinate pneumatization also called concha bullosa or bullous middle turbinate is one of the possible etiologic factors in nasal obstruction, recurrent sinusitis, and headache [5]. Bullous middle turbinate is one of the most common anatomical variations present in the nasal cavity. The incidence of BMT ranges from $14 \%$ to $53 \%$ in most studies. In our study there were cases with bullous middle turbinate's (Figures $9 \& 10$ ) but presence of bullous middle turbinates was not evaluated and reported. Patients with bullous middle turbinates were treated in cooperation with ENT specialists in our clinic.

The advent of the endoscopic diagnostic nasal examination combined with paranasal computed tomographic scanning allowed better insight into disorders of the paranasal sinuses [8]. So paranasal sinus computed tomographic scanning can detect sinus pathologies in patients who apply for aesthetic rhinoplasty. If there is reasonable suspicion for pathology in the paranasal sinuses, radiologic imaging becomes necessary and a paranasal computed tomography scan should be performed [9]. Combined rhinoplasty and endoscopic sinus surgery can be performed safely, cost-effectively and with very good results in selected patients $[8,10,11]$. Endoscopic sinus surgery should not be performed at the same time as rhinoplasty in patients with severe sinusitis $[8,10]$. Complications were minimal and aesthetic outcomes remained uncompromised in a study evaluating results of combining rhinoplasty and endoscopic sinus surgery in 72 patients [8]. In our study none of our patients who requested cosmetic nasal surgery had paranasal computed tomographic findings of chronic sinusitis requiring surgical treatment.

In many patients who request cosmetic nasal surgery there are varying degrees of septal deviation and inferior turbinate hypertrophy. For this reason detailed preoperative evaluation of nasal airways must be carried out and plastic surgeons performing cosmetic nasal operations must be trained and be competent in nasal septal and turbinate surgery to avoid nasal obstruction following surgery. Cooperation with ENT specialists in the presence of bullous middle and/or inferior turbinates and chronic sinusitis is very important for plastic surgeons to prevent patient dissatisfaction following rhinoplasty.

\section{Limitations}

The most important limitation of this study is that presence of bullous middle turbinate was not evaluated and reported. This reason was that bullous middle turbinates were managed by the help of ENT specialist during rhinoplasty. Another study may be performed to report this condition by using preoperative paranasal computed tomography scanning. The other limitation of this study is that nasal endoscopic examination was not performed prior to preoperative paranasal computed tomography. Anyway this report was written to emphasize the importance of obtaining a preoperative paranasal computed tomography scan if you are a plastic surgeon and do not have nasal endoscopic instruments at your disposal.

\section{Conclusion}

Despite the fact that radiologic imaging is usually not a standard part of the workup in patients demanding rhinoplasty, preoperative paranasal computed tomography is essential to detect the presence of concomitant pathologies like septal deformities, inferior turbinate enlargement, bullous middle turbinate and chronic sinusitis. Preoperative computed tomography is strongly advised for the preoperative evaluation of patients with crooked nose. For plastic surgeons who do not have endoscopic instruments to examine nasal cavities, preoperative nasal computed tomography is very valuable method to assess internal nasal structures.

\section{Acknowledgement}

The author thanks Suat Sedat Cüzdan, Uğur Koçer and Ragıp Özdemir who have helped in carrying out this study.

\section{References}

1. Wotman $M$, Kacker A. What are the indications for the use of computed tomography before septoplasty?. Laryngoscope. 2016; 126: 1268-1270.

2. Lee DC, Shin JH, Kim SW, Kim SW, Kim BG, Kang JM, et al. Anatomical analysis of nasal obstruction: nasal cavity of patients complaining of stuffy nose. Laryngoscope. 2013; 123: 1381-1384.

3. Lin JK, Wheatley FC, Handwerker J, Harris NJ, Wong BJ. Analyzing nasal septal deviations to develop a new classification system: a computed tomography study using MATLAB and OsiriX. JAMA Facial Plast Surg. 2014; 16: 183-187.

4. Berkiten G, Kumral TL, Saltürk Z, Atar Y, Yildirim G, Uyar Y, et al. Effect of Deviated Nasal Septum Type on Nasal Mucociliary Clearance, Olfactory Function, Quality of Life, and Efficiency of Nasal Surgery. J Craniofac Surg. 2016; 27: 1151-1155.

5. Ozdogan F, Ozel HE, Esen E, Altıparmak E, Genc S, Selcuk A. An oftenneglected area in crooked nose: middle turbinate pneumatization. Braz J Otorhinolaryngol. 2017; 83: 563-567.

6. Pittore B, Al Safi W, Jarvis SJ. Concha bullosa of the inferior turbinate: an unusual cause of nasal obstruction. Acta Otorhinolaryngol Ital. 2011; 31: 4749.

7. Jun BC, Kim SW, Kim SW, Cho JH, Park YJ, Yoon HR. Is turbinate surgery necessary when performing a septoplasty?. Eur Arch Otorhinolaryngol. 2009; 266: 975-980.

8. Mazzola RF, Felisati G. Rhinoplasty and endoscopic surgery for functional and inflammatory nasal/sinus disorders. Plast Reconstr Surg. 2005; 115 : 705-710.

9. Bast F, Weikert S, Schrom T. Significance of the sinus x-ray prior to septoplasty. HNO. 2013; 61: 651-655.

10. Rizk SS, Edelstein DR, Matarasso A. Concurrent functional endoscopic sinus surgery and rhinoplasty. Ann Plast Surg. 1997; 38: 323-329.

11. Inanli S, Sari M, Yazici MZ. The results of concurrent functional endoscopic sinus surgery and rhinoplasty. J Craniofac Surg. 2008; 19: 701-704. 